\title{
Vascular Pathology in the Extracranial Vertebral Arteries in Patients with Acute Ischemic Stroke
}
L. Bentsen ${ }^{a}$
A. Nygård ${ }^{b}$
C. Ovesen ${ }^{a}$
A. Christensen ${ }^{b}$
S. Rosenbaum ${ }^{\mathrm{a}}$
I. Havsteen ${ }^{b}$
H. Christensen ${ }^{a}$

Departments of a Neurology and ${ }^{\mathrm{b}}$ Radiology, Bispebjerg University Hospital,

Copenhagen, Denmark

\section{Key Words}

Acute ischemic stroke · Acute stroke imaging · CT angiography · Vertebral arteries .

Vascular neurology · Pathology

\section{Abstract}

Introduction: Vascular pathology in the extracranial vertebral arteries remains among the possible causes in cryptogenic stroke. However, the diagnosis is challenged by the great variety in the anatomy of the vertebral arteries, clinical symptoms and difficulties in the radiological assessments. The aim of this study was to assess the prevalence of CT angiography (CTA)-detected pathological findings in the extracranial vertebral arteries in an acute stroke population and secondly to determine the frequency of posterior pathology as probable cause in patients with otherwise cryptogenic stroke. Method: The analysis was based on 657 consecutive patients with symptoms of acute stroke and a final diagnosis of ischemic stroke or transient ischemic attack. On admission, a noncontrast CT cerebrum and CTA were performed. A senior consultant neuroradiologist, blinded to clinical data, reviewed all CTA scans systematically, assessing the four segments of the extracranial vertebral arteries. First, the frequency of pathological findings including stenosis, plaques, dissection, kinked artery and coiling was assessed. Subsequently, we explored the extent of the pathological findings that were the most plausible causes of stroke, namely either a possible dissection or a kinked artery. Results: Findings in the extracranial vertebral arteries included significant stenosis ( $0.8 \%)$, atherosclerotic plaque types (3.8\%), possible dissections (2.6\%), kinked arteries (2.6\%) and coiling (32.0\%). Eighteen patients (2.8\%) with pathological findings had an unknown cause of stroke, likely posterior symptoms and no clinical stroke symptoms from the anterior circuit. 
Of these, 3 cases were kinked arteries (0.5\%) and 15 cases (2.3\%) were possible dissections. Conclusion: We found that in approximately $3 \%$ of the study population, the most plausible cause of the cryptogenic strokes was due to a pathological finding in the posterior extracranial vertebral arteries, being either a possible dissection or a kinked artery. In general, posterior vascular pathology is not uncommon, and CTA is a useful modality in the detection of changes based on characteristics and locations of findings in the extracranial vertebral arteries. Ultrasound examination can be a useful supplementary tool in deciding the consequence of vascular findings on CTA.

(c) 2014 S. Karger AG, Base

\section{Background}

Most registries fail to identify a definite cause of stroke in up to $25-40 \%$ of the patients, the so-called cryptogenic strokes [1]. Vascular pathology in the extracranial vertebral arteries (the V0-V3 segments) remains among the possible causes of cryptogenic stroke [2], where the severity and presence of clinical symptoms in general may be highly varied and elusive.

The vascular findings in the vertebral arteries representing both pathological changes and normal variants can be described in detail based on existing well-defined imaging criteria. However, these findings remain less well characterized than similar changes in the carotid arteries in high-risk populations [3].

CT angiography (CTA), which is unhindered by the presence of bone and air, has been reported to be comparable to the gold standard digital subtraction angiography (DSA) in accuracy when mapping the arteries of the neck from its original to its more distal segments along with their corresponding vascular pathology [4-6].

Based on findings in previous studies, atherosclerosis, stenosis, dissections and kinked arteries present the most likely causes of stroke in the cervical arteries [7-10], and the pathogenesis can be subtyped using the ASCO classification based on the etiology [11]. However, the knowledge of pathological findings in the extracranial vertebral arteries as the cause of stroke remains relatively sparse.

The aim of the present study was to determine the prevalence of CTA-detected pathological findings in the posterior extracranial vertebral arteries in an acute stroke population and to determine the frequency of posterior pathology as probable cause in patients with otherwise cryptogenic stroke.

\section{Methods}

\section{Study Population}

Six hundred and fifty-seven consecutive patients with symptoms of acute stroke and a final diagnosis of ischemic stroke or transient ischemic attack were evaluated at Bispebjerg University Hospital, Copenhagen, from June 2009 until December 2011. The institution has a catchment area of 1.7 million (The Capitol Region of Denmark) on even dates, and all patients with acute stroke symptoms are evaluated by a consultant neurologist and referred to acute stroke imaging.

The patients with pathological findings in the vertebral arteries representing a potential cause of stroke were classified according to the ASCO score with which a systematic assessment was performed and the etiology of stroke was categorized into atherosclerosis (A), small vessel disease (S), cardiac source (C) or other causes (O), including unknown other cause [11]. Patients with either a more likely cause of stroke or symptoms that best could be ascribed 
Bentsen et al.: Vascular Pathology in the Extracranial Vertebral Arteries in Patients with Acute Ischemic Stroke

Table 1. Definitions of pathological findings in the extracranial vertebral arteries

\begin{tabular}{|c|c|}
\hline Finding & Definition \\
\hline Stenosis & $\begin{array}{l}\text { The extent of stenosis is defined by the following: } \\
\% \text { stenosis }=[1-(\mathrm{D}(\text { stenosis }) / \mathrm{D}(\text { normal }))] \times 100 \text {. } \\
\mathrm{D}(\text { stenosis })=\text { the diameter of the artery at the site of the most severe stenosis, and } \mathrm{D}(\text { normal })= \\
\text { the diameter of the proximal normal artery. If the proximal segment was diseased, contingency } \\
\text { sites were chosen to measure } \mathrm{D}(\text { normal): distal artery (second choice), feeding artery (third } \\
\text { choice) [12]. } \\
\text { Stenosis between } 70-99 \% \text { is defined as significant stenosis. }\end{array}$ \\
\hline Plaque & $\begin{array}{l}\text { Presence of thickening of the vessel wall and/or presence of calcification and ulceration. } \\
\text { Smooth plaque: soft matter observed at CTA in the thickened vessel surface. } \\
\text { Calcified plaque: calcification found at CTA in the thickened vessel surface. } \\
\text { Mixed plaque: both calcified and soft matter observed at CTA in the thickened vessel surface. } \\
\text { Ulcerated plaque: extension of contrast material present beyond the vascular lumen into the } \\
\text { surrounding plaque (types 1-4): } \\
\text { 1. an ulcer that points out peripendicular to the lumen } \\
\text { 2. an ulcer with a narrow neck that points out proximally and distally } \\
\text { 3. an ulcer neck proximally and points out distally } \\
\text { 4. an ulcer neck pointing out proximally [13]. }\end{array}$ \\
\hline Dissection & $\begin{array}{l}\text { A tear in the intima of the vessel wall, which can present as an occlusion due to the small } \\
\text { diameter of the vertebral artery [14] or occur as a pseudoaneurysm [15]. Dissection is also } \\
\text { defined as multiple lumenal stenosis in the extracranial vertebral artery, the so-called } \\
\text { alternating vessel caliber }[10,16,17] \text {. }\end{array}$ \\
\hline Occlusion & No visible flow bypassing [9]. \\
\hline Kinking & $\begin{array}{l}\text { Angulations of one or more segments of the internal artery associated with stenosis in the } \\
\text { affected segment [8]. }\end{array}$ \\
\hline Coiling & $\begin{array}{l}\text { Elongation and redundancy of the artery resulting in exaggerated S-shaped curvature or in a } \\
\text { circular configuration [8]. }\end{array}$ \\
\hline Dolicoectasia & $\begin{array}{l}\text { Describes an elongation, widening and tortuosity of an artery with an increased diameter of } \\
\text { both arteries }>4 \mathrm{~mm} \text { in V2 [18]. }\end{array}$ \\
\hline Fenestration & An artery with a single origin, with two parallel segments anywhere along its course $[19,20]$. \\
\hline Duplication & An artery with two origins and a variable level of fusion in the neck $[19,20]$ \\
\hline $\begin{array}{l}\text { Dominant } \\
\text { vertebral artery }\end{array}$ & $\begin{array}{l}\text { A dominant vertebral artery is defined by one of the vertebral arteries being } 2 / 3 \mathrm{~mm} \text { larger in } \\
\text { diameter compared to the opposite. }\end{array}$ \\
\hline
\end{tabular}

to the anterior circulation were excluded. The remaining patients had pathological findings in the vertebral arteries, which represented the most likely cause of stroke (table 1).

Stroke was registered as plausibly caused by pathology in the posterior extracranial vertebral arteries when neither a recognized cause of stroke nor a sign of origin in the anterior circulation was found.

Potential causes of stroke in the cervical vessels include mixed and ulcerated atherosclerotic plaques, potential dissections and a kinked artery [7-10].

\section{Radiological Imaging and Work-Up}

On admission, the imaging setup included a noncontrast CT cerebrum and a CTA from the aortic arch to the vertex. Imaging was performed using a 64-section multidetector CT (MDCT) with a noncontrast CT cerebrum $(120 \mathrm{kVp}, 500 \mathrm{mAs}, 5 \mathrm{~mm}$ slice thickness reconstruction) and a CTA (120 kVp, $295 \mathrm{mAs}$, collimation $64 \times 0.625 \mathrm{~mm}$ isotropic voxel resolution) with contrast bolus injection (Omnipaque $350 \mathrm{mg} / \mathrm{ml}, 5 \mathrm{ml} / \mathrm{s}$ ) monitored by bolus tracking in the descending aorta, scanned with fixed $3 \mathrm{~s}$ of post tracking delay and reconstructed in $0.9-\mathrm{mm}$ thick slices.

The standard procedure in patients admitted with acute stroke symptoms is neuroimaging with CTA and subsequently monitoring with telemetry. The methods to detect atherosclerosis are CT or MRI. On suspicion of small vessel disease or dissection, MRI is performed. 
Fig. 1. The posterior extracranial vertebral artery segments.

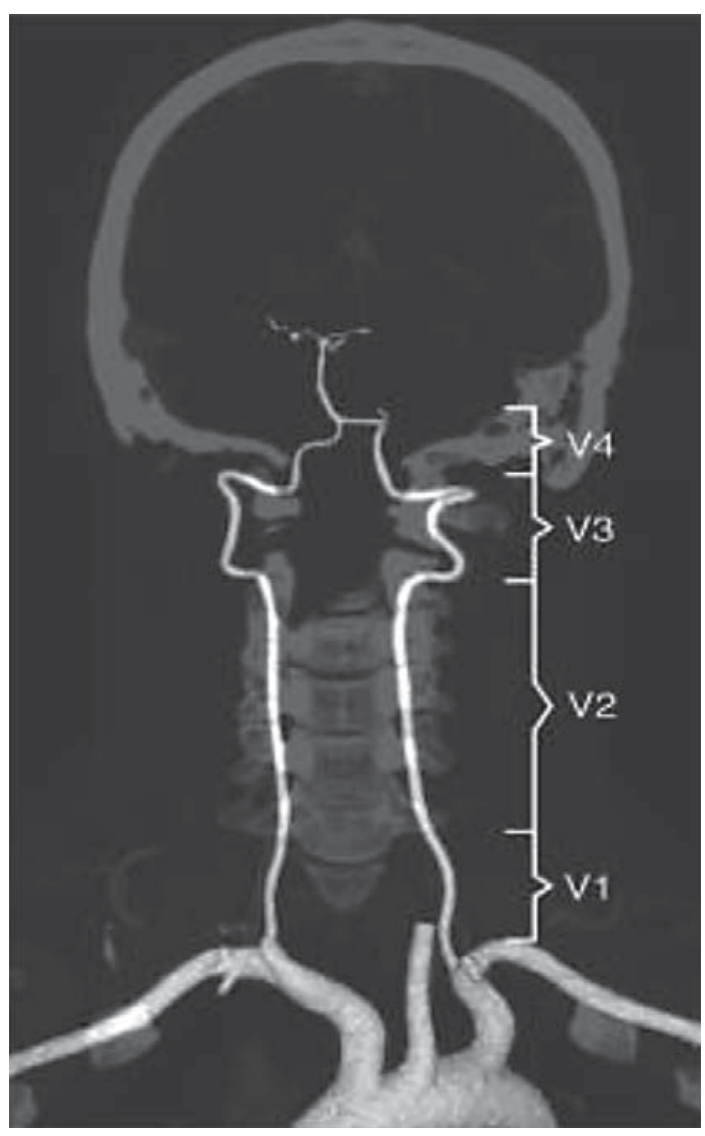

Ultrasound (US) examination with Doppler is used to quantify suspected stenosis based on CTA (mainly carotid). In case of a likely cardioembolic cause, transesophageal echocardiography and Holter monitoring (7 days) is used. In younger stroke patients with a family history of stroke, thrombophilia screening is performed.

A senior consultant neuroradiologist (A.C.) blinded to clinical data reviewed all CTA scans and systematically assessed the four segments of the vertebral arteries (fig. 1). Definitions of the pathological findings are presented in table 1.

For calculation of the frequencies of the pathological findings in the dataset, we used the statistical program SPSS, version 19.0 (IBM, Chicago, Ill., USA).

The study was approved by the Danish Data Protection Agency, file No. 2009-41-3824.

\section{Results}

\section{Distribution of Vascular Changes in Terms of Lumen Reduction}

In the segment $\mathrm{V} 0$ of the vertebral arteries, primarily the presence of stenosis and occlusions was examined. CTA imaging revealed that 9 of the 657 patients $(1.4 \%)$ had insignificant stenosis and 3 patients $(0.5 \%)$ had significant stenosis. No occlusions were observed in the V0-segment.

Stenosis was also observed in V1 in a comparable amount as seen in V0 (table 2). Only one occlusion was found in V1, while 8 occlusions involving several segments of the vertebral arteries were documented. Alternating vessel calibers were observed in 6 patients, appearing 


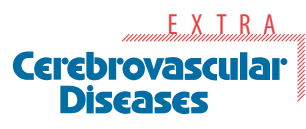

Table 2. The extent of stenosis and possible dissections in V1-V3 $(n=657)$

Table 3. Categorized stenosis plaque types $(\mathrm{n}=657)$

Table 4. Kinking versus coiling ( $\mathrm{n}=657$ )

\begin{tabular}{|c|c|c|c|c|}
\hline \multicolumn{5}{|c|}{ Cerebrovasc Dis Extra 2014;4:19-27 } \\
\hline \multicolumn{3}{|c|}{ DOI: $10.1159 / 000357663$} & \multicolumn{2}{|c|}{$\begin{array}{l}\text { (c) } 2014 \text { S. Karger AG, Basel } \\
\text { www.karger.com/cee }\end{array}$} \\
\hline \multicolumn{5}{|c|}{$\begin{array}{l}\text { Bentsen et al.: Vascular Pathology in the Extracranial Vertebral Arteries in Patients with } \\
\text { Acute Ischemic Stroke }\end{array}$} \\
\hline Segments & $<70 \%$ stenosis & $71-99 \%$ & stenosis & Possible dissection* \\
\hline V1 & $11(1.5)$ & $2(0.3)$ & & $1(0.2)$ \\
\hline V3 & - & - & & $2(0.3)$ \\
\hline $\mathrm{V} 1+\mathrm{V} 2$ & - & - & & $4(0.6)$ \\
\hline $\mathrm{V} 1+\mathrm{V} 2+\mathrm{V} 3$ & - & - & & $10(1.5)$ \\
\hline
\end{tabular}

Values are $\mathrm{n}(\%)$.

* Possible dissections are defined as occlusions or alternating vessel caliber.

\begin{tabular}{lr}
\hline Plaque type & Stenosis plaque in the vertebral arteries, $\mathrm{n}(\%)$ \\
\hline Smooth & $2(0.3)$ \\
Calcified & $12(1.8)$ \\
Mixed & $10(1.5)$ \\
Ulceration & $1(0.2)$ \\
\hline
\end{tabular}

\begin{tabular}{lcc}
\hline & Right vertebral artery & Left vertebral artery \\
\hline Kinked artery & $2(0.4)$ & $15(2.2)$ \\
In V1 & $1(0.2)$ & $14(2.1)$ \\
In V2 & $1(0.2)$ & $1(0.1)$ \\
Coiled artery & $94(14.3)$ & $116(17.6)$ \\
In V1 & $61(9.3)$ & $83(12.6)$ \\
In V2 & $6(0.9)$ & $5(0.7)$ \\
V1 + V2 & $20(3.0)$ & $21(3.2)$ \\
V1 + V2 + V3 & $7(1.1)$ & $7(1.1)$ \\
\hline
\end{tabular}

Values are $\mathrm{n}(\%)$. 
Bentsen et al.: Vascular Pathology in the Extracranial Vertebral Arteries in Patients with Acute Ischemic Stroke

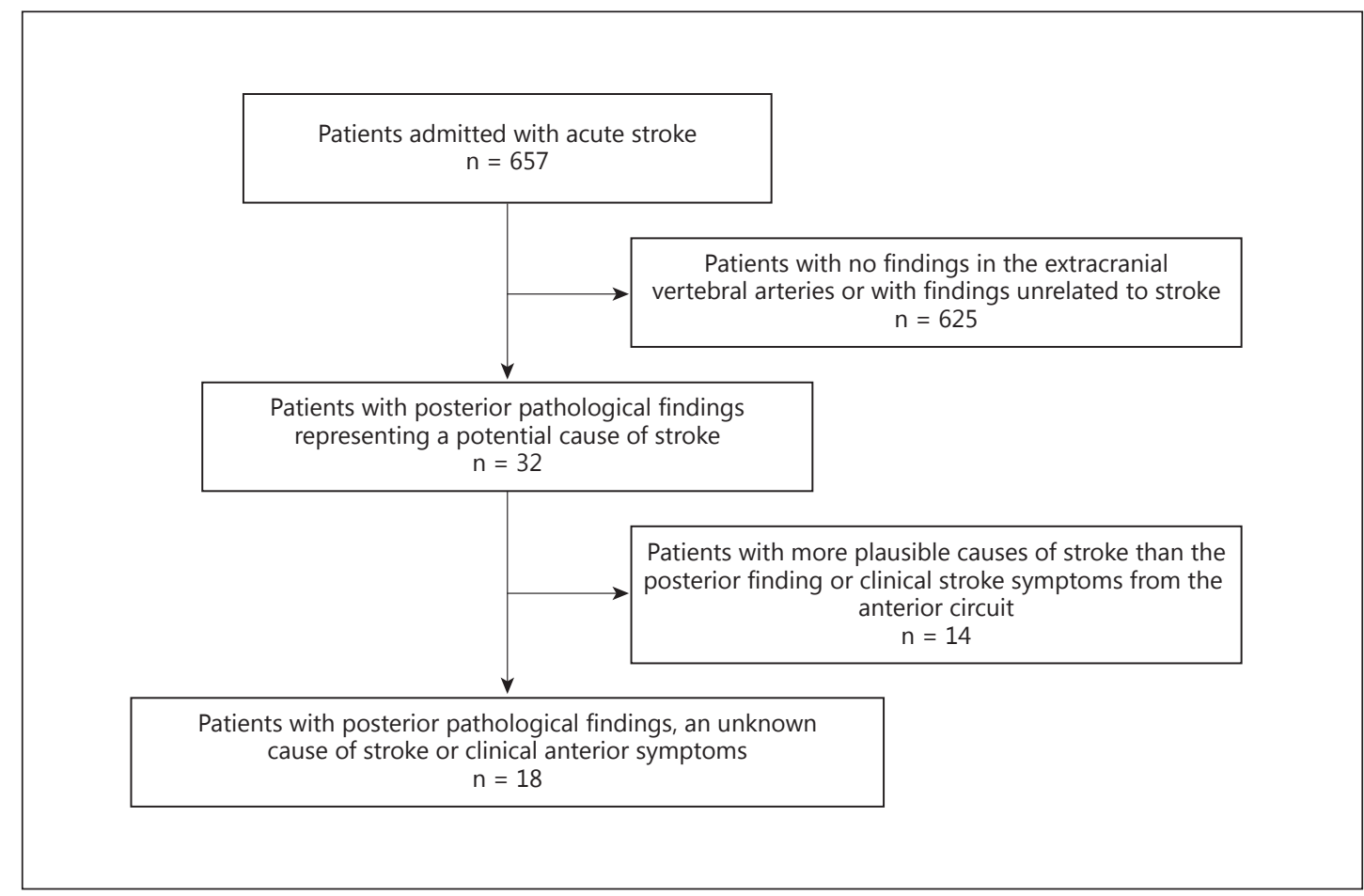

Fig. 2. Flowchart of patients with pathological findings in the extracranial vertebral arteries defined as potential cause of stroke.

\section{Dolicoectasia, Duplication and Fenestration}

In 114 patients (17.4\%), dolicoectasia was observed on CTA. In contrast, no duplication or fenestration was observed.

Pathology in the Vertebral Arteries as the Most Plausible Cause of Stroke

Thirty-two patients (4.9\%) presented pathological findings defined as potential cause of stroke (fig. 2). Eighteen of these (2.8\% of the total number of patients) had pathological findings in the vertebral arteries, which represented the most likely cause of stroke.

The most plausible cause of stroke was a kinked artery in 3 cases $(0.5 \%)$ and a presumed dissection in 15 cases (2.3\%), defined by half of the patients having an occlusion ( 7 patients) and the other half an alternating vessel caliber (8 patients).

\section{Discussion}

In the present study, dissection was by far the most frequent plausible cause of cryptogenic stroke in approximately $3 \%$, whereas the occurrence of significant stenosis and overall plaque formation was found to be minimal.

\section{Dissections}

In the present study, the overall frequency of detected possible dissections was nearly doubled compared to previous reports from stroke populations $[12,13]$. A possible cause leading to the misinterpretation of an occlusion is the presence of motion artefacts. However, 
the CT resolution is improved and motion artefacts reduced when using MDCT as a 64-slice scanner or higher. Our more frequent findings can thus be explained by a higher detection rate of changes in the vessel wall of the extracranial vertebral arteries as well as a mandatory inclusion of some patients in whom these changes can represent atherosclerotic changes. MRI can be added to separate active and nonactive changes in the vessels wall, i.e., dissection in the subacute phase in which the patients can cooperate more effortlessly to the longer scan procedures.

Segment V3 is reported as the most frequent origin of dissection in the vertebral arteries but is not the only location $[12,14,15]$. Both the $\mathrm{V} 1$ and the $\mathrm{V} 2$ segments are reported as being involved or acting as the origins of dissection $[10,16,17]$. This is in accordance with the findings of the present study, where all segments in the extracranial vertebral arteries had findings defined as dissections. This is of importance since dissection changes involving a higher number of segments increase the risk of further spread into the intradural parts of the artery, which aggravates the prognosis [14].

\section{Lumen Reductions}

In the present study, the prevalence of plausible causes of acute stroke symptoms in form of significant stenosis with or without associated unstable plaques was low in the extracranial vertebral arteries, including the origin of these arteries, i.e., the ostium and the proximal V1. This is partly in accordance with previous findings of a low prevalence of significant stenosis in this area, even in selected populations with known reduced perfusion in the posterior circuit $[7,18]$. The higher frequencies of ostial stenosis reported from other series $[17,19]$ can be attributed to the significant underestimation known to be present using CTA in comparison with DSA as the gold standard [18].

In the patients in whom lumen reduction was found to be the plausible cause of stroke, kinking was predominating. Kinking and coiling of the arteries was abundantly more frequent in the left vertebral artery compared to the right artery. No other differences were found including the lumen or other vascular changes.

The occurrence of coiled arteries and dolicoectasia in the present study population is similar to previous observations $[15,20]$. These findings represent an elongation and, in general, some degree of dilation due to degenerative changes in the vascular wall but have not been reported as cause of acute stroke symptoms [15].

This is the first study to systematically map the pathology of the extracranial vertebral arteries based on CTA in an acute stroke population. The study population consisted of consecutive stroke patients admitted to one large center based on examination by senior consultants and paramedics prior to admission, and all patients underwent a CTA that was performed within $4.5 \mathrm{~h}$ after ictus. The population is therefore unselected - except for diagnosis and timing. The same senior observer evaluated all CTA examinations and no missing values concerning clinical data were present.

A weakness of this study is the lack of a gold standard DSA to compare the CTA findings. However, the CTA performed on a MDCT with 64 slices has been validated against DSA as being able to detect small vascular changes as small as $2 \mathrm{~mm}[9,10,21]$. MRA has limited use in prolonged vascular protocols in acute stroke patients with an associated need of observation, restlessness and the risk of missing information concerning contraindications, but also because MRA covers a smaller area [22].

The findings of the present study revealed less frequent vascular changes in the most proximal part of the extracranial vertebral arteries and a more disseminated pathology in other segments, which makes Duplex US a reliable candidate for follow-up examination after the acute stroke work-up that should include a CTA [14,22]. 
Bentsen et al.: Vascular Pathology in the Extracranial Vertebral Arteries in Patients with Acute Ischemic Stroke

The problems of US visualization of the most proximal parts of the extracranial vertebral arteries are due to artefacts from bone and air, but this method is able to provide dynamic imaging of the V2 and V3 without interference. US can thus be a useful supplemental tool in deciding the consequence of vascular findings on the CTA based on the ability of US to adequately measure the flow with high resolution [3]. The US modality can thus be very helpful in mapping restricted flow due to undiscovered ostial lumen reductions and thereby indirectly herald their presence.

\section{Conclusion}

In the present study, the prevalence of different pathological findings in the posterior extracranial vertebral arteries was described in a large, single-center stroke population.

We found that in approximately $3 \%$ of the study population, the most plausible cause of the cryptogenic strokes was due to a pathological finding in the posterior extracranial vertebral arteries either because of a possible dissection or a kinked artery.

In general, posterior vascular pathology is not uncommon, and CTA is a useful modality in the detection of changes based on characteristics and locations of findings in the extracranial vertebral arteries. US can be a useful supplemental tool in deciding the consequence of vascular findings on CTA.

\section{Disclosure Statement}

The authors have no conflicts of interest to declare.

\section{References}

1 Sacco RL, Ellenberg JH, Mohr JP, Tatemichi TK, Hier DB, Price TR, et al: Infarcts of undetermined cause: the NINCDS Stroke Data Bank. Ann Neurol 1989;25:382-390.

2 Amarenco P: Underlying pathology of stroke of unknown cause (cryptogenic stroke). Cerebrovasc Dis 2009; 27(suppl 1):97-103.

3 Buckenham TM, Wright IA: Ultrasound of the extracranial vertebral artery. Br J Radiol 2004;77:15-20.

-4 Romijn M, Gratama van Andel HA, van Walderveen MA, Sprengers ME, van Rijn JC, van Rooij WJ, et al: Diagnostic accuracy of CT angiography with matched mask bone elimination for detection of intracranial aneurysms: comparison with digital subtraction angiography and 3D rotational angiography. AJNR Am Journal Neuroradiol 2008;29:134-139.

5 Papke K, Kuhl CK, Fruth M, Haupt C, Schlunz-Hendann M, Sauner D, et al: Intracranial aneurysms: role of multidetector CT angiography in diagnosis and endovascular therapy planning. Radiology 2007;244:532-540.

6 Chaudhary SR, Ko N, Dillon WP, Yu MB, Liu S, Criqui GI, et al: Prospective evaluation of multidetector-row CT angiography for the diagnosis of vasospasm following subarachnoid hemorrhage: a comparison with digital subtraction angiography. Cerebrovasc Dis 2008;25:144-150.

7 Farres MT, Grabenwoger F, Magometschnig H, Trattnig S, Heimberger K, Lammer J: Spiral CT angiography: study of stenoses and calcification at the origin of the vertebral artery. Neuroradiology 1996;38:738-743.

-8 Weibel J, Fields WS: Tortuosity, Coiling, and Kinking of the Internal Carotid Artery. II. Relationship of morphological variation to cerebrovascular insufficiency. Neurology 1965;15:462-468.

-9 Khan S, Cloud GC, Kerry S, Markus HS: Imaging of vertebral artery stenosis: a systematic review. J Neurol Neurosurg Psychiatry 2007;78:1218-1225.

10 Teasdale E, Zampakis P, Santosh C, Razvi S: Multidetector computed tomography angiography: application in vertebral artery dissection. Ann Indian Acad Neurol 2011;14:35-41.

11 Amarenco P, Bogousslavsky J, Caplan LR, Donnan GA, Hennerici MG: New approach to stroke subtyping: the A-S-C-O (phenotypic) classification of stroke. Cerebrovasc Dis 2009;27:502-508.

12 Hodler J, von Schulthess GK, Zollikofer CL: Diseases of the Brain, Head \& Neck, Spine: Springer Verlag Italia; 2008.

13 Redekop GJ: Extracranial carotid and vertebral artery dissection: a review. Can J Neurol Sci 2008;35:146-152. 
14 Flis CM, Jager HR, Sidhu PS: Carotid and vertebral artery dissections: clinical aspects, imaging features and endovascular treatment. Eur Radiol 2007;17:820-834.

15 Saba L, Mallarini G: Correlation between kinking and coiling of the carotid arteries as assessed using MDCTA with symptoms and degree of stenosis. Clin Radiol 2010;65:729-734.

16 Lleva P, Ahluwalia BS, Marks S, Sahni R, Tenner M, Risucci DA, et al: Traumatic and spontaneous carotid and vertebral artery dissection in a level 1 trauma center. J Clin Neurosci 2012;19:1112-1114.

17 Caplan L, Wityk R, Pazdera L, Chang HM, Pessin M, Dewitt L: New England Medical Center Posterior Circulation Stroke Registry II. Vascular lesions. J Clin Neurol 2005;1:31-49.

-18 Kumar Dundamadappa S, Cauley K: Vertebral Artery Ostial Stenosis: Prevalence by Digital Subtraction Angiography, MR Angiography, and CT Angiography. J Neuroimaging 2013;23:360-367.

19 Caplan LR: The intracranial vertebral artery: a neglected species. The Johann Jacob Wepfer Award 2012. Cerebrovasc Dis 2012;34:20-30.

20 Lou M, Caplan LR: Vertebrobasilar dilatative arteriopathy (dolichoectasia). Ann NY Acad Sci 2010;1184:121133.

21 Kramer M, Vairaktaris E, Nkenke E, Schlegel KA, Neukam FW, Lell M: Vascular mapping of head and neck: computed tomography angiography versus digital subtraction angiography. J Oral Maxillofac Surg 2008;66: 302-307.

22 Puchner S, Haumer M, Rand T, Reiter M, Minar E, Lammer J, et al: CTA in the detection and quantification of vertebral artery pathologies: a correlation with color Doppler sonography. Neuroradiology 2007;49:645650. 